\title{
Nouveau droit comptable: dispositions plus sévères pour les médecins
}

\author{
Depuis le $1^{\text {er }}$ janvier 2013 le nouveau droit comptable est entré en vigueur. Un mé- \\ decin qui a réalisé un chiffre d'affaires supérieur à 500000 francs a l'obligation de \\ tenir une comptabilité et de présenter des comptes.
}

\begin{abstract}
Martin Brenner $r^{a, b}$, Rolf Willimann ${ }^{a}$

a FMH Fiduciaire Services b FMH Insurance Services
\end{abstract}

\begin{abstract}
Généralités
Le 22 novembre 2012, le Conseil fédéral a communiqué que le nouveau droit comptable entrera en vigueur le $1^{\text {er }}$ janvier 2013. Un délai transitoire de deux ans est accordé aux entreprises concernées pour s'adapter à la nouvelle situation juridique. A partir de l'exercice 2015, elles devront impérativement appliquer les dispositions du nouveau droit comptable. Désormais, l'obligation de tenir une comptabilité et de présenter des comptes ne dépendra plus de la forme juridique d'une entreprise mais de son importance économique.
\end{abstract}

\section{Désormais, l'obligation de tenir une comptabilité et de présenter des comptes ne dépendra plus de la forme juridique d'une entreprise mais de son importance économique.}

Correspondance: FMH Consulting Services AG Burghöhe 1 CH-6208 Oberkirch Tél. 0419250077 Fax 0419210586

\section{Limites du chiffre d'affaires}

En vertu des nouvelles dispositions, l'obligation de tenir une comptabilité et de présenter des comptes s'applique aux:

- entreprises individuelles et sociétés de personnes qui ont réalisé un chiffre d'affaires supérieur à 500000 francs lors du dernier exercice;

- personnes morales.

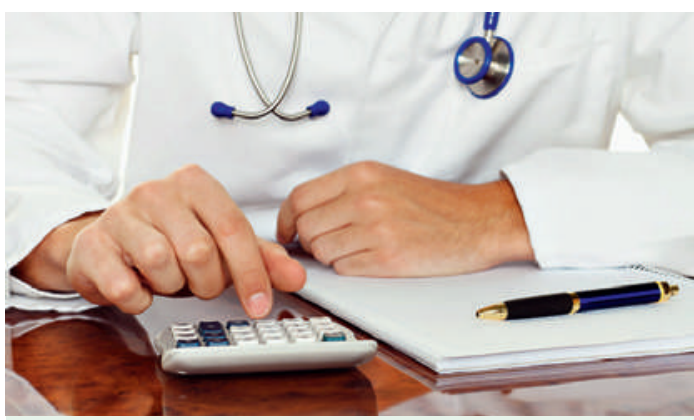

Entreprises individuelles et sociétés de personnes qui ont réalisé un chiffre d'affaires supérieur à 500000 francs ont l'obligation de tenir une comptabilité et de présenter des comptes.
Les entreprises individuelles et les sociétés de personnes qui ont réalisé un chiffre d'affaires inférieur à 500000 francs lors du dernier exercice doivent comptabiliser uniquement les recettes et les dépenses (conformément aux principes de la délimitation périodique et du rattachement des charges aux produits) ainsi que le patrimoine.

Les entreprises individuelles et les sociétés de personnes qui ont réalisé un chiffre d'affaires inférieur à 100000 francs peuvent déroger au principe de la délimitation périodique et comptabiliser uniquement les recettes et les dépenses courantes.

\section{Conséquences pour les médecins}

Les cabinets médicaux qui ne tiennent qu'une comptabilité simple (type «carnet du lait») en raison d'un chiffre d'affaires inférieur à 500000 francs doivent savoir que les autorités fiscales exigent généralement des informations supplémentaires.

Les médecins qui réalisent un chiffre d'affaires supérieur à 500000 francs ont l'obligation de tenir une comptabilité et de présenter des comptes; cette disposition légale concerne en particulier les médecins qui remettent des médicaments car ils dépassent souvent ce montant. La nouvelle situation juridique s'accompagne de dispositions plus sévères. Il faudra désormais présenter les recettes et les dépenses conformément aux principes de la délimitation périodique et du rattachement des charges aux produits.

En principe, les autorités fiscales s'en tiennent à une clôture correcte dans le cadre du droit commercial, pour autant que les dispositions fiscales complémentaires soient respectées. Par contre, les entreprises individuelles et les sociétés de personnes peuvent renoncer à établir l'annexe obligatoire avec les informations explicatives supplémentaires si elles ne sont pas soumises aux dispositions régissant l'établissement des comptes des grandes entreprises (à partir d'une somme de 20000000 francs inscrite au bilan, d'un chiffre d'affaires de 40000000 francs et de 250 postes à plein temps). Néanmoins, si les dispositions requièrent des informations supplémentaires, celles-ci doivent impérativement être indiquées dans le bilan et dans le compte d'exploitation. 